\title{
Alternative Therapy for Breast Cancer: Outcomes, Obstacles and Opportunities
}

\author{
Sheldon Marc Feldman, MD, FACS \\ Division of Breast Surgical Oncology, Columbia University Medical Center, New York City, NY
}

The use of complementary and alternative medicine (CAM) is widespread by people pursuing optimal health and well being. The 2007 National Health Interview Survey (NHIS) showed that approximately $38 \%$ of American adults use CAM. Greenlee et al. reported CAM use in $96.5 \%$ of 1,000 women diagnosed with breast cancer (BC) in northern California. ${ }^{1}$ The definition of CAM practices is quite broad and is best categorized by the National Center for Complementary and Alternative Medicine (www.nccam.nih.gov.) into the following five categories: natural products, including dietary supplements, such as botanicals, vitamins, minerals, and probiotics; mind-body medicine, including meditation, yoga, acupuncture, visualization, hypnotherapy, tai chi, and deep-breathing exercises; manipulative and body-based practices, including spinal manipulation and massage; movement therapies, including Feldenkrais method, Alexander technique, Pilates, and Trager psychophysical integration; other healing modalities, including traditional healers, manipulation of energy fields, Reikki, therapeutic touch, qi gong, magnet and light therapy, and other medical systems, including Ayurvedic and traditional Chinese medicine. Spirituality, prayer, nutritional approaches, including fasting and detoxification, music, and exercise interventions also are included by some authors as CAM usage by patients.

Han et al. report in this issue of the ASO on the outcomes of a series of 61 women with operable breast cancer who chose primarily herbal, vitamin, and dietary alternative therapies and refused or delayed recommended surgical, systemic therapy, and radiation treatments. ${ }^{2}$ This is in sharp contrast to many patients with $\mathrm{BC}$ who utilize complementary approaches in addition to accepted

(C) Society of Surgical Oncology 2011

Published Online: 21 January 2011

S. M. Feldman, MD, FACS

e-mail: sf2388@columbia.edu standard of care toward the goal of strengthening their immune system, controlling treatment-related side effects, and improving their overall health and wellness. It can be very rewarding to witness an overall improvement in quality of life among many BC survivors who energetically work to improve their lifestyle after the trauma of a breast cancer diagnosis. Dr. Han and colleagues document disease progression and increased risk of recurrence and death among the patients who declined or delayed traditional treatment. Twenty of the 26 patients who omitted or delayed surgical therapy died or progressed to stage 4 disease. This is a truly unique, contemporary series of patients with $\mathrm{BC}$ collected during a 10-year period, which demonstrates the natural history of the disease. This is an important contribution for patients who are considering alternative therapies to understand objectively the significantly adverse outcomes from such an approach. It helps to counterbalance anecdotal claims of miracle cures from alternative practitioners. It is truly remarkable that 57 of the 61 patients returned to their clinic for care when disease progression occurred. This is the result of a compassionate, respectful approach where keeping active communication open and avoiding judgement allowed alternatively oriented patients to feel safe in returning to reconsider and accept traditional care. This approach allowed 20 of Dr. Han's patients to survive without evidence of disease. This is in stark contrast to the attitude of "my way or the highway," which unfortunately is still pervasive.

There are significant obstacles to caring for patients who decline recommended treatments. These patients have been referred to as demanding, noncompliant, difficult, or somewhat euphemistically special or exceptional. Historically, there has been an element of authoritarianism in the doctor-patient relationship. These patients challenge that dynamic and may have fixed ideas about what is acceptable. They are armed with a great deal of information, often from noncredible sources, and often are quite 
passionate about "natural" healing approaches. They can be extremely demanding, time-consuming, disruptive to schedules, and insistent on immediate access to the health care team. An additional possible burden is the concern about medical-legal malpractice consequences by participating in the care of a patient who declines traditional recommended therapy. Attempting to care for such patients can be extremely anxiety-provoking and psychologically exhausting. Yet, many such patients are welcomed and accepted by alternative practitioners and develop "meaningful" healing relationships. These alternative approaches are so highly valued by some patients that they deplete financial reserves and drastically modify their lifestyle to comply with rigorous regiments, including supplements, colonics, special diets, and fasting. Unfortunately, many of these patients will not change course when their disease progresses because they may be convinced that they have not followed the regiment properly and ultimately succumb to their disease. They often experience a cruel and painful demise, which is heartbreaking.

There are a great many opportunities to learn from the experience of Dr. Han and associates toward improving patient care. The importance of communication to optimize patient care cannot be overemphasized. The alternative practitioners clearly do this more effectively. The model of "shared medical decision" making is becoming more normative and empowers patients to actively participate in their healing. ${ }^{3}$ This breaks down the authoritarian relationship and expands the patient's role in the decision process. The multidisciplinary team approach to cancer care, which includes psycho-oncology, is enormously helpful to assess the appropriateness of decision making and to help support the process. Medical schools and residency training programs now provide excellent training models to enhance physician-patient communication. ${ }^{4}$ The mindful choice of words and an empathetic attitude can be powerful tools for healing. Poorly considered language can be quite damaging to patients and often is irreversible. The Arnold Gold Foundation (www.humanism-in-medicine.org) helps to advance the core values of professionalism and humanism as vital aspects of medical training and practice. We have the opportunity to provide meaningful mentorship to trainees that transcends the operating room and can greatly impact the next generation. More patients are demanding an integrated approach to their cancer care and want guidance on the use of CAM. NCAM (nccam.nih.gov) and The Society for Integrative Oncology (www.integrativeonc.org) provide education, resources, and funding to help advance the goal of evidence-based evaluation of purported beneficial approaches. A recent example is the Institute of Medicine (IOM) report on the controversy about the supplemental use of vitamin $\mathrm{D}$ for breast cancer prevention. There have been two recent studies showing efficacy for the use of acupuncture in aromatase inhibitor-associated joint symptoms and in managing vasomotor symptoms in patients with hormone receptor-positive $\mathrm{BC} .^{5,6}$ At Columbia, we have initiated a study that incorporates the role of Reikki practitioners in applying mind-body principles and energy healing to help to prepare patients for breast cancer surgery and emotionally accept the loss of their breast(s). We must continue to strive to provide care that is patient-centered and reduces the stress and trauma of disease.

Patients are demanding a comprehensive approach to their care that transcends surgery, systemic therapy, and radiation. The $\mathrm{BC}$ multidisciplinary team has grown and in many centers includes psycho-oncology, nutrition, social worker, exercise physiologist, clergy, wellness coordinator, peer support, music therapist, and navigation. Addressing patient care in such a holistic-integrated fashion leads to a high degree of patient satisfaction, but it is critically important that each component be evaluated for efficacy, particularly in an environment that requires cost containment. We need to be open to evaluating all potential healing modalities that may be beneficial. I greatly admire the bravery and dedication of patients who work to blend the best of traditional medicine and CAM approaches. I believe that they achieve the best quality of life. We must to continue to learn from patients who chose only alternative care. We can overcome the obstacles and seize the opportunities to provide objective evaluations of risk and benefit. Dr. Han and colleagues have clearly demonstrated the adverse outcomes that result from pursuing alternative care only. Hopefully, these patients will reduce in number as we more effectively address their concerns about traditional care.

\section{REFERENCES}

1. Greenlee H, Kwan K, Ergas IJ, et al. Complementary and alternative therapy use before and after breast cancer diagnosis: the Pathways Study. Breast Cancer Res Treat. 2009;117:653-65.

2. Han E, Johnson N, DelaMelena T, et al. Alternative therapy used as primary treatment for breast cancer negatively impacts outcomes. ASO-2010-11-1750.

3. Tariman JD, Berry DL, Cochrane B, et al. Preferred and actual participation roles during health care decision making in persons with cancer: a systemic review. Ann Oncol. 2010;21(6)1145-51.

4. Bub B. Communication skills that heal: a practical approach to a new professionalism in medicine. Radcliffe Publishing, ISBN-10 1 $857756649,2006$.

5. Crew KD, Capodice JL, Greenlee H, et al. Randomized, blinded, sham-controlled trial of acupuncture for the management of aromatase inhibitor-associated joint symptoms in women with early-stage breast cancer. J Clin Oncol. 2010;28(7):1154-60.

6. Walker EM, Rodriguez AI, Kohn B, et al. Acupuncture versus venlafaxine for the management of vasomotor symptoms in patients with hormone receptor-positive breast cancer: a randomized trial. J Clin Oncol. 2010;28(4):634-40. 